\title{
Comparison of Solar Trackers and Application of a Sensor Less Dual Axis Solar Tracker
}

\author{
Ceyda Aksoy Tirmikci and Cenk Yavuz \\ Electrical and Electronics Engineering Department, Engineering Faculty, Sakarya University, Sakarya 54187, Turkey
}

Received: February 05, 2015 / Accepted: April 03, 2015 / Published: June 30, 2015.

\begin{abstract}
In the 21th century, one of the biggest problems that the world faces is energy provision. Today, the way that most countries use for energy production is not clean and continuous. The most obvious proof for that is the change in the climate. The amount of $\mathrm{CO}_{2}$ (carbon dioxide) that is caused by fossil fuel burning increases day by day and this situation creates the greenhouse effect and change in the climate. The sun is one of the best solutions to this problem. It is not only clean and continuous, but also available anywhere, anytime. However, the efficiency of a photovoltaic system is directly related to the amount of solar energy acquired by the system which means that, it is necessary to follow the sun to have a high efficient system. There are two types of trackers that follow the sun and there are several ways to build them. In this paper, solar tracker designs are examined and a new dual axis solar tracker design is given.
\end{abstract}

Key words: Solar energy, sensorless solar tracker, solar math, linear actuators, efficiency, low cost.

\section{Introduction}

Energy shortage is one of the most important problems in the world today. As the most common energy sources fossil fuels have been depleting rapidly and the greenhouse gas emission has been seriously threatening the environment, the search for an alternate source of power has been increasing. Solar power is one of the best alternative power sources since it is available in nature.

Solar power is the conversion of sunlight into electricity, either directly using PV (photovoltaic), or indirectly using CSP (concentrated solar power). Concentrated solar power systems use lenses or mirrors and tracking systems to focus a large area of sunlight into a small beam. Photovoltaic convert light into electric using the photovoltaic effect.

Photovoltaic systems are arrangements of components designed to supply usable electric power for a variety of purposes, using the sun as the power

Corresponding author: Ceyda Aksoy Tirmikci, research assistant, research fields: solar energy, energy efficiency, environmental impact and sustainability. E-mail: caksoy@sakarya.edu.tr. source. These systems are very common and used for a variety of applications. As they supply electric power using the sun, their efficiency is directly related to the amount of solar energy acquired by the system. The more solar energy acquired, the more electric power supplied. So, it is necessary to follow the sun to increase the efficiency.

A solar tracker is a device that orients a payload toward the sun. In photovoltaic applications, trackers are used to minimize the angle of incidence between the incoming sunlight and a photovoltaic panel. This increases the amount of energy produce from a fixed amount of installed power generating capacity. There are two types of solar trackers: single axis trackers and dual axis trackers.

In this study, solar tracking is examined and a dual axis solar tracker is designed according to the results of comparisons.

\section{Single Axis Trackers}

Single axis trackers have one degree of freedom that acts as an axis of rotation. The axis of rotation of single 
axis trackers is typically aligned along a true north meridian. It is possible to align them in any cardinal direction with advanced tracking algorithms.

There are several common implementations of single axis trackers. These include HSAT (horizontal single axis trackers), VSAT (vertical single axis trackers), TSAT (tilted single axis trackers) and PSAT (polar aligned single axis trackers). The orientation of the module with respect to the tracker axis is important when modeling performance.

Al-Mohammad [1] designed a single axis sun-tracking system. Two symmetric photo-resistors are used to track the sun's position. A PLC (programmable logic unit) is employed to control and monitor the mechanical movement of the PV module and collect and store data related to the sun's radiation. It is found that, the daily output power of the PV was increased by more than $20 \%$ in comparison with that of a fixed module. The PV-tracking system can be employed as a standalone device and it could be connected to a personal computer through the RS232 serial port to monitor the whole process on a computer screen.

Abdallah and Badran [2] designed a single axis sun-tracking system for enhancing solar still productivity. A computerized sun tracking device is used for rotating the solar still with the movement of the sun. In this study, the programming method of control works efficiently in all weather conditions regardless of the presence of clouds. The calculated values of the surface positions as a function of time are fed to the PLC program to control the actuator of the sun position tracker, $24 \mathrm{~V}$ AC electrical motor. A comparison between fixed and sun tracked solar stills shows that the use of sun tracking increased the productivity for around $22 \%$, due to the increase of overall efficiency by $2 \%$. It can be concluded that, the sun tracking is more effective than fixed system and it is capable of enhancing the productivity.

Sefa, et al. [3] designed a one axis solar tracking system. A DC motor with $24 \mathrm{~V}, 50 \mathrm{~W}$ is used for the movement of the solar panel in east or west directions. All movement procedures are controlled using two photo resistors. The tracking system includes a serial communication interface based on RS485 to monitor whole processes on a computer screen and to plot data as graphic. In addition, system parameters such as the current, the voltage and the panel position have been observed by means of a microcontroller. The energy collected is measured and compared with a fixed solar system for the same solar panel. The results show that, the solar energy collected on the tracking system is considerably much efficient than the fixed system.

Al-Haddad and Hassan [4] designed a one axis solar tracking system. The control part of the system is made using electronic circuit that have the op-amp LM324 as the main component. While for the mechanical part, the moving base of the reflector of the satellite receiver antenna is used. Measurements have been made for comparison between fixed and tracking system. The results have shown that, the tracking system is effective in the sense of relatively high output power increase and low cost.

Agarwal and Pal [5] designed a computer based one axis solar tracking system. LDR is used as photo sensor to sense the incident solar radiation. A computer based stepper motor is used in the tracking system to provide motion to the photovoltaic panel. The results show that, in cloudy weather, the system cannot track the actual position of the actual position of the sun, because of the absence of shading effect.

\section{Dual Axis Trackers}

Dual axis trackers have two degrees of freedom that act as axes of rotation. These axes are typically normal to one another. The axis that is fixed with respect to the ground can be considered a primary axis.

The axis that is referenced to the primary axis can be considered a secondary axis. There are several common implementations of dual axis trackers. They are classified by the orientation of their primary axes with respect to the ground. Two common 
implementations are TTDAT (tip-tilt dual axis trackers) and AADAT (azimuth-altitude dual axis trackers).

Mamlook, et al. [6] designed a two axis sun tracking system. A programmable logic controller is used to control the motion of a two axis sun tracking surface. Results are compared with those fixed at a surface tilted $32^{\circ}$ towards south. Preliminary measurements indicated that, the use of two axis sun tracking would increase daily energy collection by more than $40 \%$.

Roth, et al. [7] designed a two axis sun tracking system. The tracker gives the possibility for automatic measuring of direct solar radiation with a pyrheliometer. In the active operation mode, the tracker uses the signal of a sun detecting linear sensor to control the pointing. Two stepper motors move the instrument platform, keeping the sun's beam at the center of the sensor.

Hassan, et al. [8] designed a two axis sun tracking system. The system is sensorless and open loop controlled. A wiper motor has been used for azimuth tracking and a linear actuator has been used for declination tracking. Tested results shows that a significant increase of the efficiency of the tracker.

Duarte, et al. [9] designed a two axis sun tracking system. This work studies the solution of two axis solar tracking system based on solar maps, which can predict the exact apparent position of the sun, by the latitude's location, thereby avoiding the need to use sensors or guidance systems. To accomplish this, it is used a low-power microcontroller, suitably programmed, to control two electric motors to ensure that the panels supporting structure is always oriented towards the sun.

There are other studies of dual axis trackers with different designs. They all support the result that dual axis trackers are more efficient than single trackers [10-13].

\section{A Dual Axis Tracker Design}

In this study, a sensor less dual axis tracker is designed. The system is controlled with a low power microcontroller based on solar mathematics. Two linear actuators are used to provide the dual axis movement.

\subsection{The Sun's Apparent Position}

The earth is almost spherical in shape, some $12,700 \mathrm{~km}$ in diameter and it revolves around the sun in a slightly elliptical (almost circular) orbit. The earth-sun distance is approximately 150 million $\mathrm{km}$, varying between 152 million $\mathrm{km}$ (at aphelion, on July 01) and 147 million $\mathrm{km}$ (at perihelion, on January 01 ). The full revolution takes 365.24 days ( 365 days $5 \mathrm{~h} 48^{\prime} 46^{\prime \prime}$ to be precise) and as the calendar year is 365 days, an adjustment is necessary: one extra day every four years (the "leap year"). This would mean 0.25 days per year, which is too much. The excess 0.01 day a year is compensated by a one day adjustment per century. The plane of the earth's revolution is referred to as the ecliptic. The earth's axis of rotation is tilted $23.45^{\circ}$ from the normal to the plane of the ecliptic. The angle between the plane of the earth's equator and the ecliptic (or the earth-sun line) is the DEC (declination) and it varies between $+23.45^{\circ}$ on June 22 (northern solstice) and $-23.45^{\circ}$ on December 22.

Geographical LAT (latitude) of a point on the earth's surface is the angle subtended between the plane of the equator and the line connecting the center with the surface point considered. ALT (altitude) is measured in the vertical plane, between the sun's direction and the horizontal, in some texts, this is referred to as "elevation" or "profile angle" (Fig. 1). AZI (azimuth) is the direction of the sun measured in the horizontal plane from north in a clockwise direction and have $-90^{\circ}$ for east and $+90^{\circ}$ for west, or the converse for the southern hemisphere, taking $0^{\circ}$ for north and going through east to $+180^{\circ}$ and through west to $-180^{\circ}$ (Fig. 1) [14, 15].

There are a lot of solar angle equations. In this study, the equations below are used [14]:

- $\mathrm{DEC}=0.33281$

$-22.984 \cos (N)+3.7872 \sin (N)$ 
$-0.3499 \cos (2 N)+0.03205 \sin (2 N)$

$-0.1398 \cos (3 N)+0.07187 \sin (3 N)$

$N$ is the number of the day according to the calendar.

- $\mathrm{ALT}=\arcsin (\sin (\mathrm{DEC}) \sin (\mathrm{LAT})+\cos (\mathrm{DEC})$ $\cos ($ LAT) $\cos (\mathrm{HRA}))$

where, hour angle-HRA $=15$ (hour -12$)$.

- $\mathrm{AZI}=\arccos [(\cos (\mathrm{LAT}) \sin (\mathrm{DEC})$

$\cos (\mathrm{DEC}) \sin (\mathrm{LAT}) \cos (\mathrm{HRA})) / \cos (\mathrm{ALT})]$

Equations show that, it is enough to know the latitude value and the time of the day. As it is aimed to use the designed system anywhere in the world, changing the latitude value is enough to calculate the sun's apparent position.

\subsection{Mechanical Design}

In the mechanical design process, the system components are chosen and tested in Solidworks environment [16].

In this study, two linear actuators are used to move the solar panel in dual axis. A linear actuator creates motion in a straight line, in contrast to the circular motion of a conventional electric motor. Different types of electric motors can be used in the actuators. With the certain movement control, the actuators provide stable, quiet and good movement. They are energy efficient and long lasting without requiring periodic maintenance. The actuators used in this study have $24 \mathrm{~V} \mathrm{DC}$ motors. Their maximum thrust is $600 \mathrm{~N}$ and their maximum speed is $300 \mathrm{~mm} / \mathrm{s}$. By the limit switching properties of the actuators, the infinite movement of the motors is stopped. The only absence

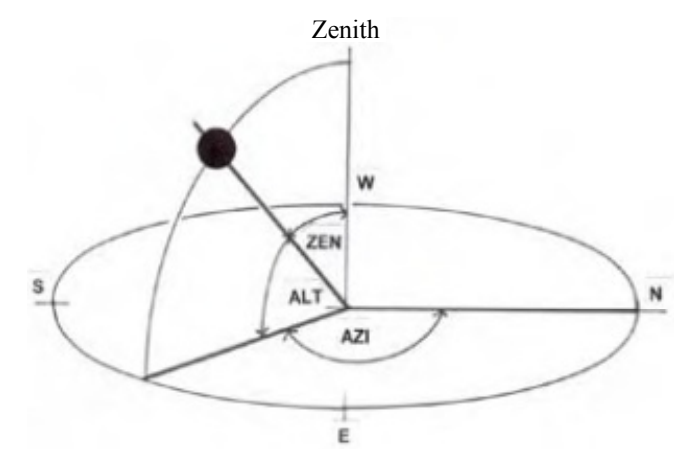

Fig. 1 Definition of solar angles. of these actuators is being lack of positional feedback. As the ones with the positional feedbacks are very expensive, the position feedback is provided in a different way.

Solar panel is chosen according to the actuators. To choose the most proper panel, the actuators are drawn in Solidworks environment and tested with different solar panels. The tests give the best movement flexibility with a $80 \mathrm{~W}, 1,185 \mathrm{~mm} \times 545 \mathrm{~mm} \times 35 \mathrm{~mm}$.

After choosing the system components, two different systems are designed in Solidworks environment. First system is a typical sun tracking system with actuators (Fig. 2). This system is tested for wind and pressure. The test results show that, the system is still stable for the wind with the speed of $36 \mathrm{~km} / \mathrm{h}$ and for the pressure of $500 \mathrm{~N} / \mathrm{m}^{2}$.

The second design is a not a typical one, it is new (Fig. 3). It is cheaper and lighter than the first design. It is also stronger that it is stable for the wind with the speed of $60 \mathrm{~km} / \mathrm{h}$, almost twofold of the first system.

\subsection{Electronic Design}

Solar tracking systems are controlled in different ways. The most common ways to control the trackers are using PLCs, microcontrollers, PCs or relays. As the

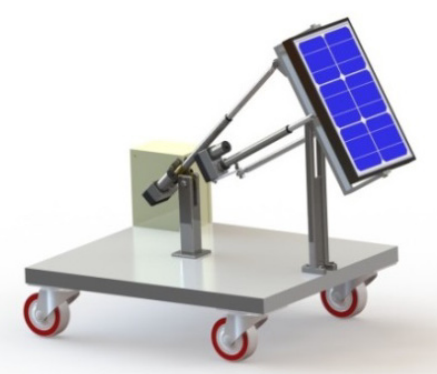

Fig. 2 The first two axes solar tracking system design.

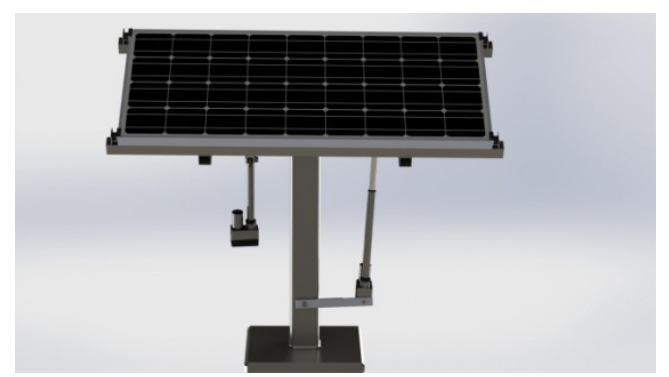

Fig. 3 The second dual axis solar tracker design. 
tracker designed in this study is proposed to be a portable and economical one, a PIC16F877A microcontroller is chosen as the control unit. PIC16F877A is cheap, small and easy to find. PIC16F877A is programmed by the hi-tech $\mathrm{C}$ compiler in the MPLAB IDE environment.

As it is mentioned in the mechanical design part, the actuators that are used in this study have $24 \mathrm{~V} \mathrm{DC}$ motors and they do not have positional feedback. The positional feedback is very important for the system to get a good efficiency. This problem is solved economically and simply by using two cheap and small linear potentiometers to get the necessary feedback. Potentiometers are placed to connection points. The potentiometers' values are measured for the positions of $0^{\circ}, 90^{\circ}, 180^{\circ}, 270^{\circ}, 360^{\circ}$ and a formula is created to translate these values into degrees in all positions of the panel accordingly. This formula is embedded to microcontroller so that it calculates the panel's position in degrees for azimuth and altitude axis.

Another problem with actuators is that PIC is not sufficient enough to drive the $24 \mathrm{~V}$ DC motors. So a L298 bridge is used to drive the motors.

Solar angle equations are given in the sun's apparent position part. To calculate these equations, it is necessary to know the time of the day. In this study, a real time clock chip, DS1302, is used to get the time of the day. In the control algorithm, the data that comes from DS1302 are taken and the date, the hour,

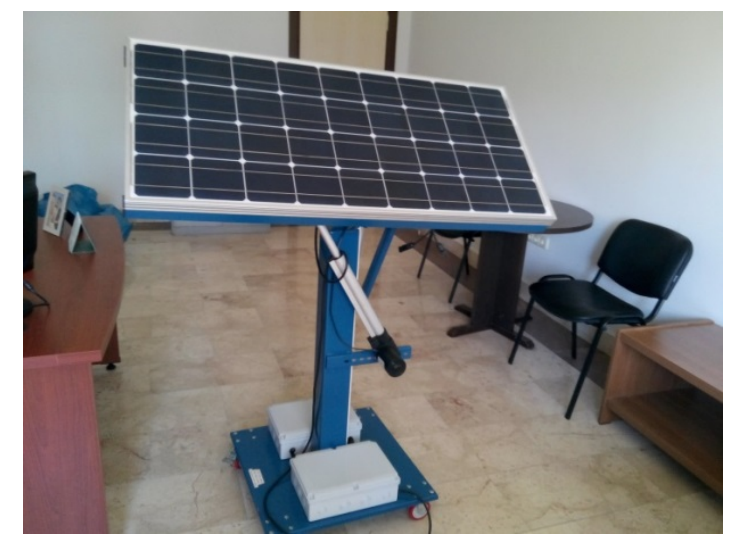

Fig. 4 Dual axis solar tracker. the minute, the second and the day number of the year are calculated. Then, these values are used to calculate the solar declination, azimuth and elevation angle. Azimuth and elevation solar angles are compared to panel's two axes positions that come from potentiometers hourly. According to the result, actuators move forward, backward or they stop. As the actuators' speed, $300 \mathrm{~mm} / \mathrm{s}$, very suitable for the dual axis movement, no speed control is done in this study.

The control algorithm is first tested in Proteus ISIS environment. In this simulation environment, the errors are found out and algorithm is corrected. After the simulation, electronic circuit is set up. Then finally the mechanic design is mounted as below (Fig. 4).

\section{Conclusions}

Comparisons of solar trackers designed show that dual axis trackers are the most efficient ones of all. In good applications, they have $40 \%$ more efficiency than fixed panels where single trackers have $20 \%$. But they have some disadvantages as well, they are more complicated and expensive than the single axis trackers.

In this study, a dual axis solar tracker is designed. The type of the tracker is chosen according to the results of past studies. It is purposed to make an economical, flexible and efficient design.

An algorithm based on solar mathematics is chosen for solar tracking. The reason of this choice is that, sensors are extra cost and their working efficiency depends on weather conditions. So the sensor less design both reduces the cost and increases the efficiency.

Microcontroller is chosen as the control unit. Microcontrollers are cheap, easy to find and very small. So they do not only reduce the cost and but also increase the flexibility.

Linear actuators are chosen to supply the movement. Linear actuators are new in solar trackers but they are easy to control and their speed is very suitable for dual axis tracking. Actuators used in this study do not have 
position feedback. This seems to be a problem at first. But this problem is solved by using cheap linear potentiometers. It makes the control algorithm a bit more complicated but helps to minimize the cost.

The main purpose of this study is to help renewable energy become more common in Turkey, where energy consumption and $\mathrm{CO}_{2}$ emission are in increasing numbers, by designing a highly efficient and low cost system which can be set up easily anywhere in the world. The next step of this study is to analyse and test the solar tracker in a year period and improve its absences.

\section{Acknowledgments}

This study was supported by the Sakarya University Scientific Research Projects Commission, Engineering Faculty and Electrical and Electronics Engineering Department.

\section{References}

[1] Al-Mohammad, A. 2004. "Efficiency Improvements of Photo-Voltaic Panels Using a Sun-Tracking System." Applied Energy 79 (3): 345-54.

[2] Abdallah, S., and Badran, O. 2008. "Sun Tracking System for Productivity Enhancement of Solar Stil." Desalination 220 (1-3): 669-76.

[3] Sefa, I., Demirtas, M., and Colak, I. 2009. “Application of One-Axis Sun Tracking System.” Energy Conversion and Management 50 (11): 2709-18.

[4] Al-Haddad, M. K., and Hassan, S. S. 2011. "Low Cost Automatic Sun Path Tracking System." Journal of Engineering 17 (1): 116-30.

[5] Agarwal, S., and Pal, S. 2011. "Design, Development and
Testing of a PC based One Axis Suntracking System for Maximum Efficiency." Sensors \& Transducers Journal 131 (8): 75-82.

[6] Mamlook, R., Nijmeh, S., and Abdallah, S. M. 2006. “A Programmable Logic Controller to Control Two Axis Sun Tracking System." Information Techonology Journal 5 (6): 1083-7.

[7] Roth, P., Georgiev, A., and Boudinov, H. 2005. "Cheap Two Axis Sun Following Device.” Energy Conversion and Management 46 (7-8): 1179-92.

[8] Hassan, I., Khan, N., and Islam, K. 2010. "Two Axis Sensorless Solar Tracking System." Presented at the NECEC (The 19th Annual Newfoundland Electrical and Computer Engineering Conference) 2010, St. John's, Newfoundland.

[9] Duarte, F., Gaspar, P. D., and Gonçalves, L. C. 2011. "Two Axes Solar Tracker based on Solar Maps Controlled by a Low-Power Microcontroller." Journal of Energy and Power Engineering 5 (7): 671-6.

[10] Eke, R., and Senturk, A. 2012. "Performance Comparison of a Double-Axis Sun Tracking Versus Fixed PV System." Solar Energy 86 (9): 2665-72.

[11] Mahmood, J. R., and Muhammed, H. 2011. "Design and Implementation of Smart Relay base Two Axis Sun Tracking System." Iraq J. Electrical and Electronic Engineering 7 (1): 259-63.

[12] Wang, J., and Lu, C. 2013. "Design and Implementation of a Sun Tracker with a Dual-Axis Single Motor for an Optical Sensor-Based Photovoltaic System." Sensors 13 (3): 3157-68. doi:10.3390/s130303157.

[13] Sungur, C. 2009. "Multi-axes Sun-Tracking System with PLC Control for Photovoltaic Panels in Turkey." Renewable Energy 34 (4): 1119-25.

[14] Szokolay, S. 2007. Solar Geometry. Queensland: PLEA (Passive and Low Energy Architecture International).

[15] Yung, C. S., and Lansing, F. L. 1982. Rates of Solar Angles for Two-Axis Concentrators. TDA Progress report.

[16] Tran, P. 2011. Solidworks 2012 Part 2: Advanced Techniques. Mission: Schroff Development Corporation. 